\title{
Ecological function according to entransy dissipation applied to the optimization of shell and tube heat exchangers
}

\author{
${ }^{1}$ Dr.C. Maida Bárbara Reyes Rodríguez, ${ }^{3}$ Dr.C. Jorge Laureano Moya Rodríguez, ${ }^{2}$ Dr. C. Oscar Miguel Cruz Fonticiella, \\ ${ }^{3}$ Dr. Jandecy Cabral Leite \\ maidab@uclv.edu.cu,jorgemoyar@gmail.com,ocf@uclv.edu.cu,jandecy.cabral@itegam.org.br \\ ${ }^{1}$ Universidad Central Marta Abreu de Las Villas, UCLV, Cuba \\ ${ }^{2}$ Centro de Estudios Energéticos y de Tecnologías Ambientales (CEETA) \\ ${ }^{3}$ Instituto de Tecnologia e Educação Galileo da Amazônia (ITEGAM)
}

\begin{abstract}
The heat exchangers of tube and shell are of the most important equipment in the industry. Its thermodynamic design is based on the global coefficient of heat transference and the total pressure drop. In 2007 a new thermodynamic property settled down denominated "Entransy", which expresses the capacity of a body to transfer heat. The loss of that capacity is denominated "Dissipation of Entransy". In order to evaluate the ecological impact of the thermal machines, Angle-Brown created in 1991 the call ecological function. In the present work the dissipation of entransy with the ecological function was combined and a new expression was created to evaluate the environmental impact of the heat exchangers. In addition the muti-objective optimization was realized to these equipment. The ecological function and the cost were used like functions objective. In order to realize the optimization the method of the Genetic Algorithms was used.
\end{abstract}

Key words: optimization, heat exchangers, ecological function, entransy, genetic algorithms.

\section{Función ecológica según la disipación de entransia aplicada a la optimización de intercambiadores de calor de tubo y coraza}

\section{RESUMEN}

Los intercambiadores de calor de tubo y coraza son de los equipos más importantes en la industria. Su diseño termodinámico se basa en el coeficiente global de transferencia de calor y la caída de presión total. En 2007 se estableció una nueva propiedad termodinámica denominada "Entransía", que expresa la capacidad de un cuerpo de transferir calor. A la pérdida de esa capacidad se le denomina "Disipación de Entransía". Para evaluar el impacto ecológico de las máquinas térmicas, Angulo-Brown creó en 1991 la llamada función ecológica. En el presente trabajo se combinó la disipación de entransía con la función ecológica y se creó una nueva expresión para evaluar el impacto ambiental de los intercambiadores de calor. Se realizó además la optimización mutiobjetivo de estos equipos. Fueron utilizadas como funciones objetivo la función ecológica y el costo. Para realizar la optimización se utilizó el método de los Algoritmos Genéticos.

Palabras Clave: optimización, intercambiadores de calor, función ecológica, entransía, algoritmos genéticos.

\section{INTRODUCCIÓN}

Ya, desde los 70’s del siglo pasado, la humanidad reconoció que el uso extensivo de los recursos naturales llevaría a su agotamiento. Unido a este hecho, la contaminación ambiental devino un problema principal para la humanidad. Como una posible solución a este hecho surgió el concepto de desarrollo sustentable o sostenible; éste fue definido por [1], como "un desarrollo que asegura las necesidades del presente de la sociedad, sin comprometer la habilidad de las futuras generaciones para satisfacer sus propias necesidades".

El uso más racional y eficaz de la energía térmica es imposible sin conocer a profundidad las leyes del intercambio de calor. Existen muchos procesos de ingeniería que requieren de la transferencia de calor. Los intercambiadores de calor (ICs) están presentes en la mayoría de los sistemas térmicos complejos de las industrias y representan el vehículo más ampliamente usado para la transferencia de calor en las aplicaciones de los procesos 
Maida Bárbara Reyes Rodríguez et al.,/ ITEGAM-JETIA Vol.01, No 04, pp.60-66. Dezembro, 2015.

industriales [2, 3]. Por muchos años, el diseño de estos equipos ha sido un gran reto para los investigadores, debido a las exigencias del ahorro energético. Dentro de ellos, los intercambiadores de calor del tipo de coraza y tubo (ICTC) constituyen la parte más importante de los equipos de transferencia de calor sin combustión en las plantas de procesos químicos y su uso es muy amplio en la producción de potencia $[4,5]$.

Para [6] en su trabajo "Modelagem de trocadores de calor casco e tubos", señalan que a pesar de la gran aplicabilidad de los intercambiadores de calor de tubo y coraza, su diseño no presenta de acuerdo a los fluidos, a las condiciones de trabajo y a los métodos empleados la precisión deseada.

Por [7] desarrollaron en el año 2003 un programa de computación para el diseño de intercambiadores de calor donde se examinan casi todas las alternativas posibles de configuración del intercambiador de tubo y coraza. No se optimiza ningún parámetro y solamente abarca fluidos monofásicos y está concebido para uno y dos pases por el tubo.

Los Algoritmos Genéticos (GAs) se desarrollaron mayormente en los años 70s como una herramienta de optimización, aunque ya se había realizado algún trabajo previo en el campo de la computación evolutiva. En 1967, [8] introdujo las palabras "algoritmo genético" y publicó la primera aplicación de los GAs.

Inspirado en el principio de la mínima generación de entropía propuesto por [9][10;11] desarrolló el enfoque de la minimización de la generación de entropía (EGM).

No obstante el enfoque de la minimización de la generación de entropía ampliamente aplicado a la modelación y optimización de sistemas térmicos que deben su imperfección termodinámica a la transferencia de calor y masa y a las irreversibilidades debidas al flujo de fluidos muestra algunas inconsistencias y paradojas cuando se aplica al diseño de intercambiadores de calor [12]. Esto se debe a que el centro del enfoque de la minimización de la generación de entropía se basa en los procesos de conversión de calor en trabajo, mientras que en el diseño de los intercambiadores de calor lo más importante es la velocidad y la eficacia de la transferencia de calor.

Por analogía con la conducción eléctrica, en el año 2007 [13] definieron un nuevo concepto físico denominado Entransy, que describe la habilidad de un cuerpo o sistema para transferir calor. Esta cantidad, que será referida como "Entransía" $\left(E_{h}=\frac{1}{2} Q_{\text {vh }} \cdot T\right)$, corresponde a la energía eléctrica almacenada en un capacitor (ver Tabla A-6.1 del Anexo A-6). Los análisis de transferencia de calor demuestran que la Entransía de un objeto describe su capacidad de transferencia de calor, de la misma forma que la energía eléctrica en un capacitor describe su capacidad de transferencia de carga eléctrica. La disipación de Entransía ocurre durante procesos de transferencia de calor como una medida de la irreversibilidad de la transferencia de calor.

Los conceptos de entransía y disipación de entransía fueron utilizados para desarrollar el principio de la disipación de entransía para la optimización de los procesos de transferencia de calor. Para un flujo de calor en una frontera fija, el proceso de conducción es óptimo cuando se reduce al mínimo la disipación de entransía [14]. Lo [15] hacen un resumen de los trabajos publicados entre el 2003 y el 2010 sobre laaplicación de la entransía a los procesos de transferencia de calor.

\section{FUNCIÓN ECOLÓGICA}

La ecología industrial fue popularizada por Frosch y Gallopoulos [16] que se preguntaron: ¿por qué los sistemas industriales no se comportan como un ecosistema, donde los desechos de algunas especies son los recursos para otras especies? ¿Por qué no deben las salidas de una industria ser las entradas de otra, reduciendo de tal modo el uso de materias primas y de la contaminación, y ahorrando en el tratamiento de desechos? Lowe y Evans [17] observan que la ecología industrial sugiere usar el diseño de ecosistemas para dirigir el reajuste o rediseño de los sistemas industriales para alcanzar un mejor equilibrio entre el funcionamiento industrial y los apremios ecológicos y por lo tanto para determinar una trayectoria al desarrollo sostenible. Según este concepto, las tecnologías industriales modernas se deben diseñar como los ecosistemas donde:

(i) se reducen al mínimo los flujos de entrada de masa y de energía

(ii) el suministro de energía es proporcionado por fuentes de energía renovable.

La minimización del consumo de energía de combustibles fósiles en procesos industriales implica la eliminación de los flujos de salida de energía inútil o de emisión de desechos al ambiente.

El análisis y la optimización de los ciclos termodinámicos ha sido uno de los aspectos más importantes y más analizados en la teoría de la Termodinámica de tiempo finito. A partir de la potencia de salida y de la tasa de generación de entropía, se presentó por primera vez un nuevo criterio para evaluar motores térmicos en el año 1991 por [18]:

$$
E^{*}=P-T_{e^{*}} \sigma
$$

Donde $\mathrm{P}$ es la potencia de salida del motor, Tc es la temperatura del reservorio frio y $\sigma$ es la tasa de generación de entropía.

Como la función objetivo $E^{*}$ es semejante, en cierto sentido, al objetivo ecológico, entonces esta función también se conoce como función objetivo ecológica.

La autora del presente trabajo, atendiendo a la paradoja de la generación de entropía y a la función de Angulo-Brown, desarrolló, por analogía a esta función, una nueva función ecológica para el caso de los intercambiadores de calor: $\mathrm{E}_{\mathrm{E}}{ }^{\prime}$, basada en la disipación de entransía.

La nueva función desarrollada basada en la función de Angulo-Brown es la siguiente:

$$
\mathrm{E}_{\mathrm{E}}^{\prime}=\mathrm{Q}-\frac{\Delta \mathrm{G}_{\mathrm{T}}}{\mathrm{AMTD}}
$$

donde:

AMTD es la media aritmética de la diferencia de temperatura que es la diferencia de temperatura equivalente del ICTC.

$\Delta G_{T^{*}}$ La disipación total de entransía dada en $\mathrm{kW}^{\circ} \mathrm{C}$ 
Esta expresión tiene la ventaja de tener en cuenta la disipación de entransía que se produce en el intercambiador de calor y que es la principal afectación al medio ambiente, ya que da una medida de las irreversibilidades en el proceso de transferencia de calor. De la expresión anterior se puede comprobar que para un intercambiador de calor, desde el punto de vista ecológico, es muy importante que para una determinada carga térmica, la disipación total de entransía sea mínima.

La expresión (2) se utilizó como función objetivo en la optimización de un ICTC combinándola con la minimización del costo total.

\section{II.1 ENTRANSÍA}

De acuerdo con la definición de entransía, la disipación de entransía causada por la conducción de calor en el intercambiador de calor se puede expresar como sigue [19]:

$$
\begin{aligned}
& G_{\Delta T}=-\int_{i}^{0}\left(\dot{m} c_{p} T d T\right)_{h, c}=\frac{1}{2}\left(\dot{m} c_{p}\right)_{h}\left(T_{h i}^{2}-T_{h 0}{ }^{2}\right)+ \\
& \frac{1}{2}\left(\dot{m} c_{p}\right)_{c}\left(T_{c i}^{2}-T_{c o}^{2}\right)
\end{aligned}
$$

Para el Estudio de Caso que se va a considerar como el fluido caliente es vapor saturado la ecuación para la disipación de entransía por conducción quedaría como sigue:

$G_{\Delta T}=-\int_{i}^{0}\left(\dot{m} c_{p} T d T\right)_{h, c}=(\dot{m})_{h} \lambda T_{V}+\frac{1}{2}\left(\dot{m} c_{p}\right)_{c}\left(T^{2}{ }_{c i}-T^{2}{ }_{c 0}\right)$

o

$G_{\Delta T}=-\int_{i}^{0}\left(\dot{m} c_{p} T d T\right)_{h, c}=(\dot{m})_{h} \lambda T_{v}-\frac{1}{2} * U_{\text {ocal }} * A_{T} * L M T D *$ $\left(T_{c i}+T_{c 0}\right)$

donde: $\lambda$ es el calor latente de cambio de fase del vapor $\left(\mathrm{h}_{\mathrm{g}}-\mathrm{h}_{\mathrm{f}}\right)$, $\mathrm{y}$ Tv es la temperatura de saturación del vapor. Por otro lado la ecuación para la disipación de entrnsía por fricción quedaría:

$$
\begin{aligned}
& G_{\Delta P}=-\int_{i}^{0}\left(\frac{m \tau}{\rho} d P\right)_{h, c}=\left(\dot{m} \frac{\Delta P}{\rho} \frac{\left(\tau_{0}-\tau_{\nu}\right.}{\ln \left(\frac{T_{\rho i}}{\tau_{e \theta}}\right)}\right)_{h, c}= \\
& \dot{m}_{v} P_{v} \bar{v} T_{v} \ln \left(\frac{P_{o}}{P_{i}}\right)+\dot{m}_{s} \frac{\Delta P_{s}}{\rho_{s}} \frac{\left(\tau_{c e}-\tau_{c i}\right)}{\operatorname{in} \frac{T_{c e}}{T_{e i}}}
\end{aligned}
$$

donde: $\dot{m}_{V}, P_{V}$ "y" $\bar{v}$ son el flujo másico, la presión y el volumen específico del vapor saturado, $P i$ coincide con $P v$ "y"

$$
P o=P i-\Delta P t
$$

Las ecuaciones (5) y (6) también son nuevas expresiones desarrolladas por la autora para este caso particular en que el fluido caliente está cambiando de fase. El primer sumando del miembro derecho de la ecuación (6) se dedujo a partir de una expresión dada para gases ideales en [20].

La disipación total de entransía dada en $\mathrm{kW}{ }^{\circ} \mathrm{C}$ puede ser obtenida sumando la disipación de entransía debido a la conducción de calor y la de entransía causada por la fricción del fluido como sigue:

$$
\Delta G_{T}=G_{\Delta T}+G_{\Delta P}
$$

$$
\begin{aligned}
& \Delta G_{T}=\frac{1}{2} U_{0 \text { cal }} * A_{T} * F * L M T D\left[\left(T_{\text {hi }}+T_{\text {ho }}\right)-\left(T_{\text {ci }}+T_{\text {co }}\right)\right]+\dot{m}_{\mathrm{t}} \frac{\Delta P_{\mathrm{t}}}{\rho_{\mathrm{t}}} \frac{\left(T_{\mathrm{ho}}-T_{\mathrm{hi}}\right)}{\ln \frac{T_{\text {ho }}}{T_{\mathrm{hi}}}}+\cdots \\
& \ldots m_{g} \frac{\Delta P_{s}}{\rho_{s}} \frac{\left(T_{c D}-T_{c i}\right)}{\ln \frac{T_{c D}}{T_{c i}}}
\end{aligned}
$$

\section{II.2 COSTO TOTAL}

El costo total $\mathrm{C}_{\text {tot }}$ incluye la inversión de capital (Ci) y el costo total de descuento de operación (Cod) [21].

$$
\mathrm{C}_{\text {tot }}=\mathrm{C}_{\mathrm{i}}+\mathrm{C}_{\mathrm{od}}
$$

Para el Estudio de Caso considerado, según se reporta en [22] se utilizó para el costo de inversión la siguiente expresión:

$$
\mathrm{C}_{\mathrm{i}}=\mathrm{PC} \cdot \delta_{\mathrm{M}} \cdot \delta_{\mathrm{p}} \cdot \delta_{\mathrm{T}}
$$

De la ecuación anterior PC es el costo de inversión o capital de inversión el cual depende del área de transferencia de calor.

$$
P C=3,28 \times 10^{4}\left(\frac{A_{T}}{80}\right)^{0,68}
$$

Donde $\mathrm{A}_{T}$ es el área de transferencia de calor en $\mathrm{m}^{2}$. El factor $\delta_{\mathrm{M}}$ es un coeficiente de corrección que tiene en cuenta el material que se empleará en la construcción del equipo, $\delta_{\mathrm{p}}$ tiene en cuenta la presión de trabajo del equipo y $\delta_{\mathrm{T}}$ la temperatura de operación.

En la Tabla 1 se muestran valores prácticos de los coeficientes antes mencionados.

El costo de operación relacionado con la potencia consumida en el bombeo para vencer las pérdidas friccionales se determina de las siguientes expresiones:

$$
C_{o d}=\sum_{k=1}^{n y} \frac{C_{0}}{(1+\hat{1})^{k}} \quad C_{0}=P C_{E} H
$$

$C_{E}$ es el costo de la energía eléctrica en $\$ / \mathrm{kW} h, i$ la tasa de descuento anual, ny el número de años de vida de la instalación y $H$ las horas de trabajo al año. 
Maida Bárbara Reyes Rodríguez et al.,/ ITEGAM-JETIA Vol.01, No 04, pp.60-66. Dezembro, 2015.

Tabla 1: Factores de corrección para el costo inicial [22].

\begin{tabular}{|c|c|}
\hline Material para la construcción & Factor de corrección $\delta_{\mathrm{M}}$ \\
\hline Tubos y coraza de acero al carbono & 1 \\
\hline Coraza de acero al carbono y tubos de aluminio & 1,3 \\
\hline Coraza de acero al carbono y tubos de aleación níquel-cobre & 2,1 \\
\hline Coraza de acero al carbono y tubos de acero inoxidable & 1,7 \\
\hline Tubos y coraza de acero inoxidable & 2,9 \\
\hline Presión de diseño $(\mathrm{Pa})$ & Factor de corrección $\delta_{p}$ \\
\hline 1000 & 2 \\
\hline 10000 & 1,3 \\
\hline $20000-700000$ & 1 \\
\hline 5000000 & 1,5 \\
\hline 10000000 & 1,9 \\
\hline Temperatura de diseño $\left({ }^{\circ} \mathrm{C}\right)$ & Factor de corrección $\delta_{T}$ \\
\hline $0-100$ & 1 \\
\hline 300 & 1,6 \\
\hline 500 & 2,1 \\
\hline
\end{tabular}

P es la potencia de bombeo y se determina:

$$
P=\frac{1}{w}\left(\frac{m_{t}}{\rho_{\mathrm{t}}} \Delta P_{\mathrm{t}}+\frac{m_{x}}{\rho_{x}} \Delta P_{s}\right)
$$

Donde $\eta$ es la eficiencia de bombeo (entre 0,5 y 0,7 ), $m_{t}$ y $m_{g}$ son los flujos másicos en el tubo y la coraza, $\Delta P_{t}$ es la caida de presión en los tubos, $\Delta P_{g}$ es la caída de presión en la coraza, $\rho_{t}$ es la densidad del fluido que va por el tubo y $\rho_{s}$ es la densidad del fluido que va por la coraza.

\section{ESTUDIO DE CASO}

Optimizar el diseño de un ICTC, tipo AEU de la norma TEMA para calentar $1.44 \mathrm{~kg} / \mathrm{s}$ de fueloil desde 95 hasta $130{ }^{\circ} \mathrm{C}$ a $0.62 \mathrm{~m} / \mathrm{s}$ con vapor saturado a $135^{\circ} \mathrm{C}$, estas son las condiciones actuales de operación, que se diferencian un tanto de las reportada en la hoja de datos original del equipo (este equipo se encuentra funcionando en el grupo electrógeno K-V 110 F.O de Santa Clara).

En la tabla 2 se muestran los parámetros de diseño y de operación del intercambiador real en funcionamiento, en la primera fila los valores se corresponden con la hoja de datos original y en la segunda fila los valores se corresponden con las condiciones de diseño reales existentes en la actualidad.

Para todos los resultados expuestos en las tablas sucesivas, la nomenclatura utilizada fue la siguiente: Lt- longitud del tubo, do-diámetro exterior del tubo, Lb-espaciamiento entre deflectores, Ds-diámetro interior de la coraza, Nt-número de tubos, Uo-Coeficiente total de transferencia de calor, Q-flujo de calor, Tco-temperatura de salida del fluido frío, Tho-temperatura de salida del fluido caliente, Acal-área de transferencia de calor calculada, VLn-velocidad lineal del fluido por los tubos, F. Ecol- función ecológica, Ci-costo fijo, CoD-costo de operación, $\Delta$ Pt-caída de presión en los tubos, $\Delta$ Pc-caída de presión en la coraza.

Tabla 2: Datos de diseño y de operación del ICTC real en funcionamento.

\begin{tabular}{|c|c|c|c|c|c|c|c|c|c|c|}
\hline $\begin{array}{c}\mathrm{Lt} \\
(\mathrm{m})\end{array}$ & $\begin{array}{c}\mathrm{do} \\
(\mathrm{mm})\end{array}$ & $\begin{array}{c}\mathrm{Lb} \\
(\mathrm{mm})\end{array}$ & $\begin{array}{c}\mathrm{Ds} \\
(\mathrm{mm})\end{array}$ & $\mathrm{Nt}$ & $\begin{array}{c}\mathrm{U}_{0} \\
\mathrm{~W} / \mathrm{m}^{20} \mathrm{C}\end{array}$ & $\begin{array}{c}\mathrm{Q} \\
(\mathrm{kW})\end{array}$ & $\begin{array}{c}\mathrm{Tc}_{0} \\
\left({ }^{0} \mathrm{C}\right)\end{array}$ & $\begin{array}{c}\mathrm{Th}_{0} \\
\left({ }^{0} \mathrm{C}\right)\end{array}$ & $\begin{array}{c}\text { Acal } \\
\mathrm{m}^{2}\end{array}$ & $\begin{array}{c}\text { Vel. Lin. } \\
(\mathrm{m} / \mathrm{s})\end{array}$ \\
\hline 1.4 & 10 & 60 & 210 & 184 & 734 & 188.9 & 155 & 170 & 8.5 & 4.8 \\
\hline 1.4 & 10 & 60 & 210 & 184 & 536 & 139.5 & 130 & 135 & 8.5 & 4.8 \\
\hline
\end{tabular}




\section{III.1 OPTIMIZACIÓN MULTI-OBJETIVO DEL ICTC}

Primeramente se optimiza el ICTC con los parámetros de diseño

fijos que tiene el equipo, según se muestra en la tabla 3 , donde las primeras dos columnas son: función ecológica en $\mathrm{kW}$ y costo total en $\$$.

Tabla 3: Optimización con los datos de diseño y de operación del ICTC real en funcionamiento

\begin{tabular}{|c|c|c|c|c|c|c|c|c|c|c|c|c|c|c|c|c|c|}
\hline 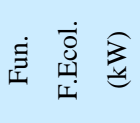 & $\begin{array}{c}\text { Costo } \\
(\$)\end{array}$ & $\begin{array}{l}\mathrm{Lt} \\
(\mathrm{m})\end{array}$ & 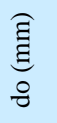 & $\mathrm{Nt}$ & $\begin{array}{l}\text { ह્ } \\
\text { ఏ }\end{array}$ & $\begin{array}{l}\text { है } \\
\hat{\text { है }}\end{array}$ & $\stackrel{?}{Z}$ & $\underbrace{\frac{3}{3}}$ & $\begin{array}{l}\Delta \mathrm{Pt} \\
(\mathrm{Pa})\end{array}$ & $\begin{array}{l}\Delta \mathrm{Pc} \\
(\mathrm{Pa})\end{array}$ & $\frac{\hat{y}}{0}$ & $\begin{array}{l}\text { Tco } \\
\left({ }^{0} \mathrm{C}\right)\end{array}$ & $\begin{array}{l}\text { Tho } \\
\left({ }^{0} \mathrm{C}\right)\end{array}$ & $\begin{array}{l}\text { Acal } \\
\left(\mathrm{m}^{2}\right)\end{array}$ & $\begin{array}{l}C_{-} \mathrm{i} \\
(\$)\end{array}$ & $\begin{array}{c}\mathrm{CoD} \\
(\$)\end{array}$ & 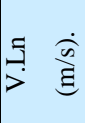 \\
\hline 59,36 & 9841 & 1,4 & 9,5 & 184 & 60 & 200 & 2,1 & 466 & 5356 & 41925 & 142 & 129,3 & 135,0 & 7,7 & 8266 & 1575 & 5,26 \\
\hline
\end{tabular}

En la figura 1 se tiene el frente de Pareto para las funciones Función Ecológica contra Costo total.

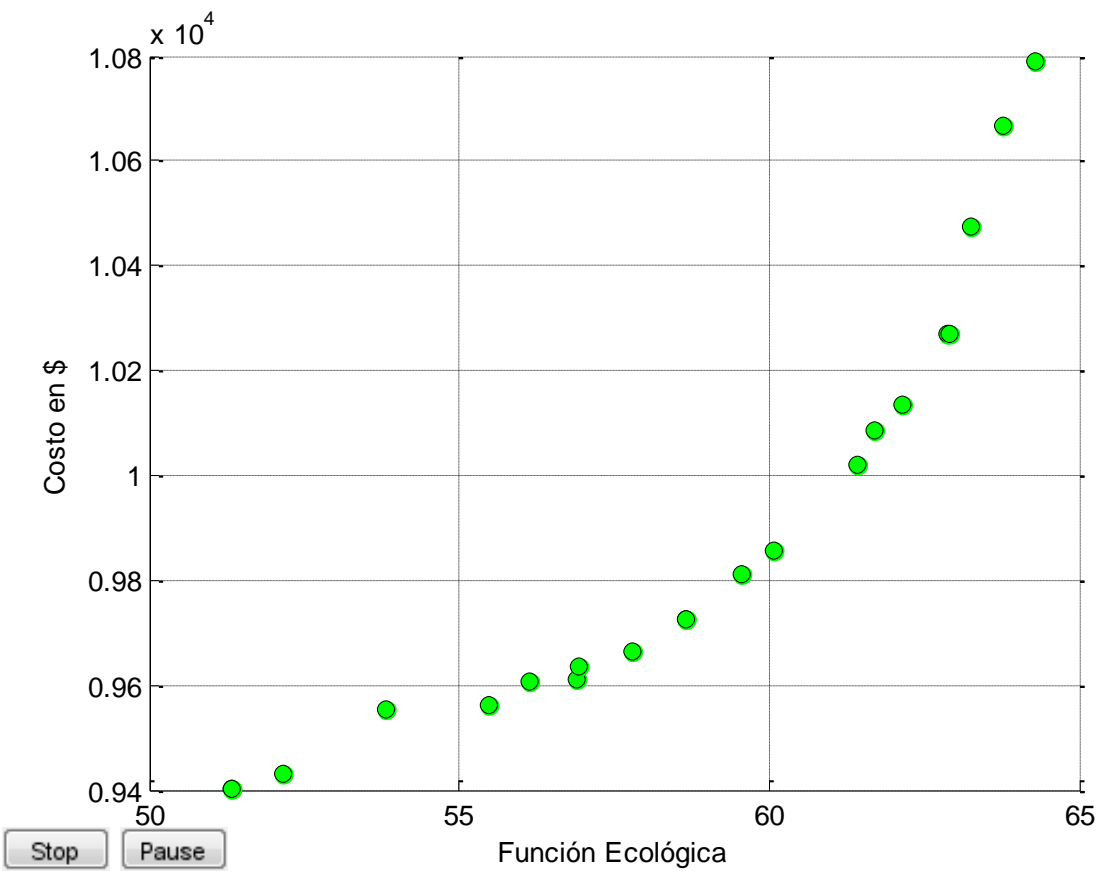

Figura 1: Función Ecológica contra Costo total.

Se realiza entonces la optimización multiobjetivo con el procedimiento propuesto. Los mejores diseños se seleccionan según los siguientes criterios:

1. El valor o los valores más cercanos a la dominancia de Pareto que resulta ser la diagonal al gráfico que parte del óptimo inalcanzable (en lo adelante criterio 1).
2. El valor que da la temperatura de salida recomendada para el fluido que se enfría o se calienta (en lo adelante criterio 2).

Como se observa en la figura anterior para lograr valores altos de la función ecológica se incrementan los costos totales. En la taba 4 se observan los mejores valores optimizados, las filas 1 y 2 según el criterio 1 y las filas 3 y 4 según el criterio 2 . 
Maida Bárbara Reyes Rodríguez et al.,/ ITEGAM-JETIA Vol.01, No 04, pp.60-66. Dezembro, 2015.

Tabla 4: Mejores diseños optimizados de Función Ecológica contra Costo total.

\begin{tabular}{|c|c|c|c|c|c|c|c|c|c|c|c|c|c|c|c|c|c|c|}
\hline$\stackrel{\imath}{z}$ & $\begin{array}{l}\text { Fun } \\
\text { Ecol. }\end{array}$ & $\begin{array}{l}\text { Costo } \\
(\$)\end{array}$ & $\begin{array}{c}\mathrm{Lt} \\
(\mathrm{m})\end{array}$ & $\begin{array}{c}\text { do } \\
\text { (mm) }\end{array}$ & $\mathrm{Nt}$ & $\begin{array}{c}\mathrm{Lb} \\
(\mathrm{mm})\end{array}$ & $\begin{array}{c}\text { Ds } \\
(\mathrm{mm})\end{array}$ & $\stackrel{?}{Z}$ & $\stackrel{\circ}{3}$ & $\begin{array}{l}\Delta \mathrm{Pt} \\
(\mathrm{Pa})\end{array}$ & $\begin{array}{l}\Delta \mathrm{Pc} \\
(\mathrm{Pa})\end{array}$ & $\begin{array}{c}\mathrm{Q} \\
(\mathrm{kW})\end{array}$ & $\begin{array}{l}\text { Tco } \\
\left({ }^{0} \mathrm{C}\right)\end{array}$ & $\begin{array}{l}\text { Tho } \\
\left({ }^{0} \mathrm{C}\right)\end{array}$ & $\begin{array}{l}\text { Acal } \\
\left(\mathrm{m}^{2}\right)\end{array}$ & $\begin{array}{l}\mathrm{C}_{-} \mathrm{i} \\
(\$)\end{array}$ & $\begin{array}{c}\mathrm{CoD} \\
(\$)\end{array}$ & $\begin{array}{l}\text { V. Ln } \\
(\mathrm{m} / \mathrm{s})\end{array}$ \\
\hline 1 & 58,65 & 9726 & 1,46 & 9,5 & 154 & 55,4 & 185 & 2 & 508 & 7645,9 & 58364,5 & 139,7 & 128,8 & 135 & 6,8 & 8265,1 & 1460,9 & 6,28 \\
\hline 2 & 60,06 & 9858 & 1,5 & 9,5 & 164 & 53,9 & 190 & 2,2 & 509 & 6741,9 & 60812,7 & 143,4 & 129,8 & 135 & 7,4 & 8265,6 & 1592,4 & 5,90 \\
\hline
\end{tabular}

\section{IV.ANÁLISIS DE LOS RESULTADOS}

Los resultados obtenidos como los mejores diseños son bastantes cercanos al existente, dado por las restricciones geométricas impuestas por el espacio disponible para instalar un posible nuevo diseño, por otra parte, según se muestra en la tabla iii si la temperatura de salida del fueloil se baja en $1.2{ }^{\circ} \mathrm{C}$, lo cual no afectaría el buen funcionamiento de los motores, se consigue con los diseños propuestos un ahorro en el costo total de $115 \$$ lo cual puede parecer un pequeño ahorro, pero si se tiene en cuenta la cantidad de intercambiadores de este tipo instalados tanto en el país como a nivel mundial, el ahorro es apreciable. Por otra parte, el uso del software propuesto en la Empresa Eléctrica donde se encuentra instalado el ICTC ha permitido una mejor operación del equipo, variando los parámetros de operación hasta seleccionar en cada momento de la operación (según las condiciones reales del vapor de calentamiento), los puntos de operación más eficientes y económicos.

\section{CONCLUSIONES}

A medida que se minimiza la tasa de disipación de entransía, se maximizan los valores de la función ecológica y se obtienen mayores valores de costo total por lo que se escogen soluciones de compromiso.

Se utilizó para la optimización multi-objetivo el NSGA II implementado en MATLAB con algunas modificaciones realizadas por la autora. El frente de Pareto obtenido en la optimización multi-objetivo permite encontrar y hacer coincidir diseños óptimos ajustables al espacio disponible para instalar el equipo y a los servicios auxiliares disponibles, sopesando todos los factores como soluciones de compromiso para lograr con un costo razonable el mejor funcionamiento térmico de equipo.

Los resultados obtenidos de la optimización multiobjetivo muestran valores muy consistentes e interrelacionados, lo que permitió seleccionar los mejores resultados del diseño optimizado. Cuando se maximiza la función ecológica con la expresión desarrollada en el trabajo, se observa que un intercambiador de calor de tubo y coraza es un equipo que no tiene un fuerte impacto ambiental, sin embargo es posible ahorrar recursos con un mejor diseño para un mismo propósito.

\section{REFERENCIAS BIBLIOGRÁFICAS}

[1] Brundtland, G. H. et al.; "Our common future", World Commission on Environment and Development, WCED, 1987.
[2] http://thermex.co.uk/Shell-and-Tube-Heat-Exchangers-1. Consultado el 27 de marzo de 2013.

[3] http://www.southwestthermal.com/shell-tube-exchanger.html. Consultado el 27 de marzo de 2013.

[4] Szargut, J.;"Exergy Method, Technical and Ecological Applications", WIT Press, Southampton; Boston, 2005, ISBN: 1-85312-753-1.

[5] Szargut, J. Stanek Wojciech., "Influence of the Proecological Tax on the Market process of fuels and electricity", ECOS 2006, Crete, 11-14 July, 2006.

[6] Balbueno Bicca, Gerson; Resende Secchi; Argimiro y Wada, Keiko."Modelagem de trocadores de calor casco e tubos". Universidade Federal do Rio Grande do Sul Seminário do Programa de Pós-Graduação em Engenharia Química. Oktober Fórum 2005 - PPGEQ.

[7] Kara Yusuf, Ali y Güraras, Özbilen., "A computer program for designing of shell-and-tube heat exchangers". Applied Thermal Engineering $\mathrm{N}^{\circ} 24$ Año 2004 paginas 1797 a 1805. Editorial Elsevier. ISSN 1369-4311.

[8] BagleyJ.D., "The behavior of adaptive systems which employ genetic and correlation algorithms", Ph.D. thesis, University of Michigan, Ann Arbor, 1967.

[9] Prigogine, I., "Introduction to Thermodynamics of Irreversible Processes", Third ed., Wiley, 1967, New York, pp.76-77.

[10] Bejan, A., "Entropy Generation through Heat andFluid Flow", Wiley,1982, New York, pp.118-134.

[11] Bejan, A., "Entropy Generation Minimization", CRCPress, 1996, Florida, pp. 47-112.

[12] Hesselgreaves, J. E.;"Rationalisation of second law analysis of exchangers," International journal of heat and mass transfer, 2000,Editorial Elsevier, 43, pp. 4159-4204.

[13] Guo, Zeng-Yuan; Zhu, Hong-Ye; Liang, Xin-Gang., "Entransy-A physical quantity describing heat transfer ability". International Journal of Heat and Mass Transfer, " 'torial Elsevier, 50 (2007) 2545-2556. , ISSN 0017-9310. 
[14] Wang Wen Hua, Cheng Xue Tao and Liang Xin Gang; "Entransy dissipation and irreversibility of some thermodynamic processes"; Chinese Science Bulletin; Engineering Thermophysics; November 2012 Vol.57 No.31: 4091-4099.

[15] Chen,Qun; Liang,Xin-Gang; Guo; Zeng-Yuan; "Entransy theory for the optimization of heat transfer $-A$ review and update"; International Journal of Heat and Mass Transfer 63 (2013) 65-81.

[16] Frosch, R.A.; Gallopoulos, N.E. (1989) "Strategies for Manufacturing" Scientific American 261:3, pp 144- 152.

[17] Lowe, E. A., Evans, L. K. Industrial ecology and industrial ecosystems. Journal of Cleaner Productions. v. 1/2, n.3, p. 47-53, 1995. apud: LAMBERT, A. J. D., BOONS, F. A.

[18] Angulo-Brown F. An ecological optimization criterion for finite-time heat engines. J Appl Phys, 1991, 69(11): 7465 7469.

[19] Guo, Jiangfeng; Li, Mengxun; Xu, Mingtian; Cheng, Lin., "The application of Entransy Dissipation Theory in optimization design of heat exchanger". Proceedings of the 14th International Heat Transfer Conference IHTC14 August 813, 2010, Washington D. C., USA.

[20] Mingtian Xu; "The thermodynamic basis of entransy and entransy dissipation"; Energy 36 (2011) 4272-4277.

[21] Taal,M.; Klemes,J.; Stehlik,P.; Cost estimation and energy price forecast for economic evaluation of retrofit projects. Applied Thermal Engineering,23.2003. Editorial Elsevier, ISSN 1369-4311.

[22] Wildi-Tremblay, Philippe and Gosselin, Louis; "Minimizing shell-and-tube heat exchanger cost with genetic algorithms and considering maintenance"; International Journal of Energy Research; 2007; 31:867-885. 